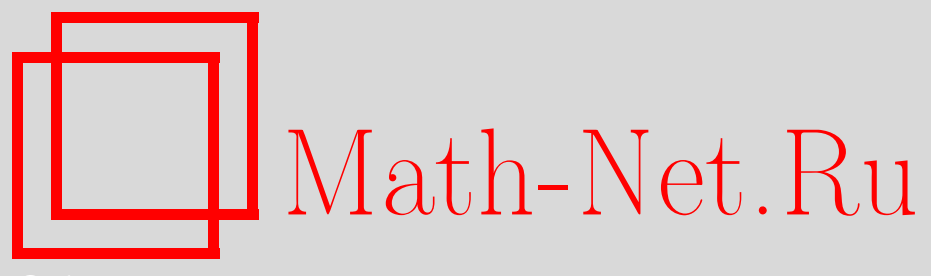

С. Г. Колесников, О рациональности и 2-рефлексивности сплетений конечных групп, Матем. заметки, 2006, том 80, выпуск 3, 395-402

DOI: https://doi.org/10.4213/mzm2825

Использование Общероссийского математического портала Math-Net.Ru подразумевает, что вы прочитали и согласны с пользовательским соглашением http://www . mathnet.ru/rus/agreement

Параметры загрузки:

IP: 3.91 .87 .62

26 апреля 2023 г., 16:08:28

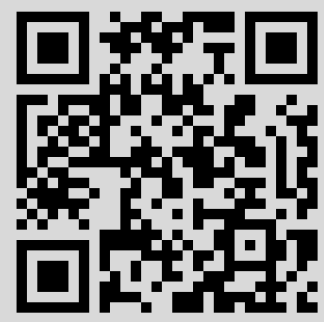




\section{О РАЦИОНАЛЬНОСТИ И 2-РЕФЛЕКСИВНОСТИ СПЛЕТЕНИЙ КОНЕЧНЫХ ГРУПП}

\section{С. Г. Колесников}

Конечная группа $G$ называется рациональной, если каждый ее неприводимый комплексный характер принимает только рациональные значения, и 2-рефлексивной, если каждый ее элемент разлагается в произведение не более двух инволюций. В статье найдены необходимые и достаточные условия рациональности и 2-рефлексивности сплетения двух конечных групп. А именно, показано, что сплетение $H$ 乙 $K$ двух конечных групп $H$ и $K$ является рациональной (аналогично, 2-рефлексивной) группой тогда и только тогда, когда $H$ - рациональная (соответственно, 2-рефлексивная) группа, а $K$ - элементарная абелева 2-группа. Как следствие получено описание классических линейных групп над конечными полями нечетной характеристики с рациональными и 2-рефлексивными силовскими 2-подгруппами.

Библиография: 9 названий.

Введение. Группы, значения всех комплексных неприводимых характеров которых рациональны (следуя Я.Г. Берковичу [1; вопрос 15.25], будем называть их рациональными группами), стали привлекать к себе интерес, прежде всего, в связи с выявившейся рациональностью симметрических групп и, более общо, групп Вейля (см., например, обзоры Ж.-П. Серра [2] и А. И. Кострикина, И. А. Чубарова [3], там же отмечается роль рациональных групп). Отвечая на вопрос Берковича [1; вопрос 15.25], автор в [4] доказал рациональность силовских 2-подгрупп симметрических групп $S_{2^{n}}$. А как известно (см., например, [5; § 5.9]), силовская 2-подгруппа группы $S_{2^{n}}$ изоморфна группе вида $\left(\ldots\left(\left(Z_{2} \succ Z_{2}\right) \succ Z_{2}\right) \ldots\right)$ 乙 $Z_{2} \quad\left(Z_{2}\right.$ встречается $n$ раз). Поэтому естественно возникает следующий

Вопрос. Каким необходимым и достаточным условиям должны удовлетворять конечные неединичные группы $H$ и $K$, чтобы их сплетение $H$ 乙 $K$ было рациональной группой?

Полный ответ на поставленный вопрос содержится в теореме 1 настоящей статьи. Оказывается, для рациональности $H$ ح $K$ необходимо и достаточно, чтобы группа $H$ сама была рациональной, а группа $K$ была элементарной абелевой 2-группой.

Работа выполнена при поддержке Российского фонда фундаментальных исследований, грант № 03-01-00905, и гранта Президента РФ № MK-1133.2005.1.

(C) С. Г. Колесников, 2006 
Отметим, что вопросом о том, какие свойства групп $H$ и $K$ переносятся на их сплетение $H$ 々 $K$, посвящен ряд работ разных авторов. В частности, Баумслагом в [6] найдены необходимые и достаточные условия нильпотентности сплетения двух групп, а в работе В.Н. Ремесленникова [7] найдены необходимые и достаточные условия, при выполнении которых дискретное сплетение двух финитно аппроксимируемых относительно сопряженности групп (ФАС-групп) остается ФАС-группой.

Группа $G$ называется 2-рефлексивной, если каждый ее элемент раскладывается в произведение не более, чем двух инволюций (элементов порядка 2) группы $G$. Интерес к 2-рефлексивным группам поддерживается как рядом известных нерешенных вопросов (в частности, вопросом об описании конечных простых 2-рефлексивных групп [1; вопрос 14.42]), так и их геометрическими приложениями. Например, в [8] 2-рефлексивные группы возникают при характеризации различных геометрий их группами движений. В п. 2 устанавливается аналог теоремы 1 для 2-рефлексивных групп. Более точно, доказывается, что группа $H$ 乙 $K$ является 2-рефлексивной тогда и только тогда, когда группа $H$ является 2-рефлексивной, а $K$ - элементарной абелевой 2-группой.

В п. 3, как следствие полученных результатов, приводится описание классических линейных групп над конечными полями нечетной характеристики с рациональными и 2-рефлексивными силовскими 2-подгруппами.

1. Теорема о рациональности сплетения групп. Доказывается следующая

Теорема 1. Сплетение $H$ \ $K$ двух конечных неединичных групп $H$ и $K$ является рациональной группой тогда и только тогда, когда $H$ - рачиональная группа, a $K$ - элементарная абелева 2-группа.

Прежде чем приступить к доказательству теоремы, напомним необходимые определения и свойства сплетений и сформулируем один критерий рациональности характеров группы.

Пусть заданы две группы $H$ и $K$. Обозначим через $H^{K}$ полное прямое произведение семейства групп $H^{k}, k \in K$, изоморфных группе $H$. Зафиксируем изоморфизмы между $H$ и $H^{k}, k \in K$, и будем обозначать через $h^{k}$ элемент из $H^{k}$, соответствующий $h \in H$. Всякий элемент $k_{0} \in K$ определяет автоморфизм группы $H^{K}$, действующий по правилу: $\phi_{k_{0}}\left(h^{k}\right)=h^{k k_{0}}$ для всех $h \in H$ и $k \in K$. Следовательно, определено полупрямое произведение $G=H^{K} \lambda K$. Полученная таким образом группа $G$ называется сплетением группы $H$ с группой $K$ и обозначается через $H$ 乙 $K$.

Пусть теперь $H$ и $K$ - конечные группы и $G=H$ 乙 $K$. Обозначим через $s$ порядок группы $K$ и пусть $k_{1}, k_{2}, \ldots, k_{s}$ - множество всех ее элементов. Каждый элемент прямого произведения

$$
H^{k_{1}} \times H^{k_{2}} \times \cdots \times H^{k_{s}}
$$

допускает единственное представление $h_{1}^{k_{1}} h_{2}^{k_{2}} \cdots h_{s}^{k_{s}}$, где $h_{1}, h_{2}, \ldots, h_{s} \in H$. Группа $K$ действует точно сопряжениями на прямом произведении (1) и поэтому каждый элемент $g \in G$ единственным образом представим в виде

$$
g=k \cdot h_{1}^{k_{1}} h_{2}^{k_{2}} \cdots h_{s}^{k_{s}}, \quad \text { где } \quad k \in K, \quad h_{1}, h_{2}, \ldots, h_{s} \in H .
$$


Разложение (2) будем называть каноническим разложением элемента $g$.

Отметим, что группу $G$ можно определить внутренним образом. А именно, группа $G$ является сплетением двух своих подгрупп $H$ и $K$, если выполняются следующие условия:

1) $H \cap K=1$ и группа $G$ порождается подгруппами $H$ и $K$;

2) элементы подгрупп $H^{k}$ и $H^{k^{\prime}}$ попарно перестановочны и $H^{k} \cap H^{k^{\prime}}=1$, если $k, k^{\prime} \in K$ и $k \neq k^{\prime}$.

Ниже мы, часто без ссылок, будем использовать свойства 1) и 2).

Также в доказательстве теоремы мы будем использовать следующий критерий рациональности характеров группы (см., например, [2; с. 61]).

ЛЕмма 1. Пусть $G$ - произвольная конечная группа и $g \in G$. Следующие условия эквивалентнъ:

1) каждыц̌ характер $G$ принимает на g рациональное значение;

2) если целое число $i$ взаимно просто с порядком элемента $g$, то элементы $g$ и $g^{i}$ сопряжены в $G$.

ДокАЗАТЕЛЬСТво теоремы 1. Предположим, что группа $G=H$ 乙 $K$ рациональна. Нам необходимо показать, что группа $H$ тоже рациональна, а $K$ является элементарной абелевой 2-группой.

Сначала докажем рациональность $H$. Обозначим через $s$ порядок группы $K$ и пусть $k_{1}, k_{2}, \ldots, k_{s}$ - множество всех элементов группы $K$. Возьмем неединичный элемент $h \in H$ и число $n$ взаимно простое с порядком элемента $h$. Так как $G$ является рациональной группой, найдется такой элемент $x \in G$, что $h^{x}=h^{n}$. Пусть

$$
x=k_{i} \cdot x_{1}^{k_{1}} x_{2}^{k_{2}} \cdots x_{s}^{k_{s}}, \quad \text { где } \quad x_{1}, x_{2}, \ldots, x_{s} \in H,
$$

- каноническое разложение элемента $x$. Тогда

$$
h^{x}=\left(x_{1}^{-1}\right)^{k_{1}} \cdots\left(x_{s}^{-1}\right)^{k_{s}} k_{i}^{-1} h k_{i} x_{1}^{k_{1}} \cdots x_{s}^{k_{s}}=\left(x_{1}^{-1} x_{1}\right)^{k_{1}} \cdots\left(x_{i}^{-1} h x_{i}\right)^{k_{i}} \cdots\left(x_{s}^{-1} x_{s}\right)^{k_{s}} .
$$

Из равенства $h^{x}=h^{n}$ и единственности разложения (2) следует, что $k_{i}=1$ и $x_{i}^{-1} h x_{i}=h^{n}$. Значит, $H$ является рациональной группой.

Докажем теперь, что $K$ является элементарной абелевой 2-группой. Предположим противное, пусть $k \in K$ и $|k|=l>2$. Рассмотрим элемент $g \in G$ вида $g=k \cdot h$, где $h$ - фиксированный неединичный элемент из $H$. Обозначим через $n$ порядок элемента $g$. Числа $n$ и $n-1$ взаимно просты, значит, ввиду рациональности $G$, элементы $g$ и $g^{n-1}=g^{-1}$ сопряжены в $G$ при помощи некоторого элемента $x$. Запишем элемент $x$ в каноническом виде $x=b \cdot x_{1}^{k_{1}} x_{2}^{k_{2}} \cdots x_{s}^{k_{s}}$, где $b \in K$ и $x_{i} \in H$. Не ограничивая общности, можно считать, что $k_{1}=k, k_{2}=k^{2}, \ldots, k_{l}=k^{l}$. Тогда

$$
g^{x}=\left(x_{1}^{-1}\right)^{k} \cdots\left(x_{l}^{-1}\right)^{k^{l}} \cdots\left(x_{s}^{-1}\right)^{k_{s}} b^{-1} \cdot k h \cdot b x_{1}^{k} \cdots x_{l}^{k^{l}} \cdots x_{s}^{k_{s}}
$$

или

$$
g^{x}=k^{b}\left(x_{1}^{-1}\right)^{k k^{b}} \cdots\left(x_{l}^{-1}\right)^{k^{l} k^{b}} \cdots\left(x_{s}^{-1}\right)^{k_{s} k^{b}} \cdot h^{b} \cdot x_{1}^{k} \cdots x_{l}^{k^{l}} \cdots x_{s}^{k_{s}} .
$$


С другой стороны,

$$
g^{x}=g^{-1}=h^{-1} k^{-1}=k^{-1}\left(h^{-1}\right)^{k^{-1}} .
$$

Сравнивая правые части равенств (3) и (4), получаем, что $k^{b}=k^{-1}$ и, значит, $b \notin\langle k\rangle$, поскольку $k \neq k^{-1}$, а также

$$
x_{1}^{-1} x_{l}=1, \quad x_{2}^{-1} x_{1}=1, \quad x_{3}^{-1} x_{2}=1, \quad \ldots, \quad x_{l-1}^{-1} x_{l-2}=1, \quad x_{l}^{-1} x_{l-1}=h^{-1} .
$$

Перемножив левые части равенств (5) справа друг на друга в обратном порядке, получим

$$
\left(x_{l}^{-1} x_{l-1}\right)\left(x_{l-1}^{-1} x_{l-2}\right) \cdots\left(x_{3}^{-1} x_{2}\right)\left(x_{2}^{-1} x_{1}\right)\left(x_{1}^{-1} x_{l}\right)=1 .
$$

В то же время, произведение правых частей равенств (5), взятых в любом порядке, равно $h^{-1}$. Значит, $h^{-1}=1$. Но $h \neq 1$. Полученное противоречие показывает, что $|k| \leqslant 2$ и, следовательно, $K$ является элементарной абелевой 2-группой.

Докажем обратное утверждение. Предположим, что группа $H$ является рациональной, а $K$ - элементарной абелевой 2-группой. В силу леммы 1 для доказательства рациональности $G$ нам достаточно показать, что любой элемент $g$ из $G$ сопряжен с любой своей степенью взаимно простой с порядком $g$. Рассмотрим два возможных случая.

Случай 1 . Пусть $g=h_{1}^{k_{1}} h_{2}^{k_{2}} \cdots h_{s}^{k_{s}}$, где $h_{1}, h_{2}, \ldots, h_{s} \in H$ и, как и выше, $k_{1}, \ldots, k_{s}-$ множество всех различных элементов группы $K$. Элементы $h_{1}^{k_{1}}, \ldots, h_{s}^{k_{s}}$ попарно перестановочны, поэтому $g^{n}=\left(h_{1}^{n}\right)^{k_{1}}\left(h_{2}^{n}\right)^{k_{2}} \cdots\left(h_{s}^{n}\right)^{k_{s}}$ для любого целого числа $n$. Пусть НОД $(n,|g|)=1$. Тогда НОД $\left(n,\left|h_{i}\right|\right)=1$ для всех $i, 1 \leqslant i \leqslant s$. Так как группа $H$ является рациональной, существует набор элементов $x_{1}, x_{2}, \ldots, x_{s}$ из $H$ таких, что $x_{i}^{-1} h_{i} x_{i}=h_{i}^{n}$ для всех $i$. Положим $x=x_{1}^{k_{1}} x_{2}^{k_{2}} \cdots x_{s}^{k_{s}}$. Ясно, что $g^{x}=g^{n}$.

Случай 2. Сначала предположим, что $|K|=2$. Пусть $h, f \in H, K=\langle k\rangle$ и пусть $g=k \cdot h f^{k}$. Докажем равенства

$$
\begin{aligned}
g^{2 n} & =(f h)^{n}\left((h f)^{n}\right)^{k}, \\
g^{2 n+1} & =k \cdot\left((h f)^{n} h\right)\left((f h)^{n} f\right)^{k}
\end{aligned}
$$

для любого натурального числа $n$. Справедливость (6) и (7) при $n=0$ очевидна. Индукция и следующие соотношения:

$$
\begin{aligned}
g^{2 n+2} & =g^{2 n} \cdot g \cdot g=(f h)^{n}\left((h f)^{n}\right)^{k} \cdot\left(k \cdot h f^{k}\right) \cdot\left(k \cdot h f^{k}\right) \\
& =k^{2} \cdot(f h)^{n}\left((h f)^{n}\right)^{k} \cdot\left(h^{k} f\right) \cdot\left(h f^{k}\right) \\
& =\left((f h)^{n} f h\right)\left((h f)^{n} h f\right)^{k}=(f h)^{n+1}\left((h f)^{n+1}\right)^{k}, \\
g^{2 n+3} & =g^{2 n+1} \cdot g \cdot g=k \cdot\left((h f)^{n} h\right)\left((f h)^{n} f\right)^{k} \cdot\left(k \cdot h f^{k}\right) \cdot\left(k \cdot h f^{k}\right) \\
& =k^{3} \cdot\left((h f)^{n} h\right)\left((f h)^{n} f\right)^{k} \cdot\left(h^{k} f\right) \cdot\left(h f^{k}\right)=k \cdot\left((h f)^{n} h f h\right)\left((f h)^{n} f h f\right)^{k} \\
& =k \cdot\left((h f)^{n+1} h\right)\left((f h)^{n+1} f\right)^{k}
\end{aligned}
$$

показывают, что равенства (6) и (7) верны для любого натурального $n$. Из (6) и (7) следует, что $g$ имеет четный порядок, а порядки элементов $h f$ и $f h$ делят порядок $g$. 
Обозначим через $m$ целое положительное число, взаимно простое с порядком элемента $g$. Тогда $m=2 n+1$ для некоторого целого $n$. Элемент $h f$ лежит в $H$ и его порядок взаимно прост с $m$. Так как группа $H$ рациональна, найдется такой элемент $b \in H$, что $b^{-1} h f b=(h f)^{2 n+1}$. Положим

$$
a=h^{-1} b(h f)^{n} h .
$$

Тогда

$$
\begin{aligned}
a^{-1} & =h^{-1}(h f)^{-n} b^{-1} h=h^{-1}(h f)^{-n} b^{-1} h f b b^{-1} f^{-1} \\
& =h^{-1}(h f)^{-n}(h f)^{2 n+1} b^{-1} f^{-1}=h^{-1}(h f)^{n+1} b^{-1} f^{-1}=(f h)^{n} f b^{-1} f^{-1} .
\end{aligned}
$$

Таким образом,

$$
a^{-1}=(f h)^{n} f b^{-1} f^{-1}
$$

Из равенств (8) и (9) следует, что

$$
\begin{aligned}
& b^{-1} h a=(h f)^{n} h, \\
& a^{-1} f b=(f h)^{n} f .
\end{aligned}
$$

Положим $x=a b^{k}$. Используя соотношения (10) и (11), получаем

$$
\begin{aligned}
g^{x} & =\left(a^{-1}\left(b^{-1}\right)^{k}\right) \cdot\left(k \cdot h f^{k}\right) \cdot\left(a b^{k}\right)=\left(k \cdot\left(a^{-1}\right)^{k} b^{-1}\right) \cdot\left(h f^{k}\right) \cdot\left(a b^{k}\right) \\
& =k \cdot\left(b^{-1} h a\right)\left(a^{-1} f b\right)^{k}=k \cdot\left((h f)^{n} h\right)\left((f h)^{n} f\right)^{k}=g^{2 n+1}=g^{m} .
\end{aligned}
$$

Пусть теперь $K$ имеет произвольный порядок $s=2^{n}$. Покажем, что этот случай сводится к рассмотренному выше. Действительно, пусть $k_{1}, \ldots, k_{s}$ - различные элементы группы $K, k$ - неединичный элемент из $K, h_{1}, \ldots, h_{s} \in H$ и $g=k \cdot h_{1}^{k_{1}} \cdots h_{s}^{k_{s}}$. Выберем в каждом смежном классе группы $K$ по подгруппе $\langle k\rangle$ по одному представителю. Будем считать, переупорядочив, если нужно, элементы $k_{i}$, что это $k_{1}, \ldots, k_{m}$, где $m=s / 2$, и $k_{m+i}=k_{i} k$ для всех $i, 1 \leqslant i \leqslant m$. Тогда элемент $g$ можно представить так: $g=k \cdot\left(h_{1}^{k_{1}} h_{m+1}^{k_{1} k}\right) \cdots\left(h_{m}^{k_{m}} h_{2 m}^{k_{m} k}\right)$. Далее рассуждения проводятся аналогично тому, как мы проводили их для произведения $h f^{k}$, только теперь их следует применить к каждому из произведений $h_{i}^{k_{i}} h_{m+i}^{k_{i} k}$. Теорема доказана.

2. Теорема о 2-рефлексивности сплетения групп. Следующая теорема является аналогом теоремы 1 для 2-рефлексивных групп.

Теорема 2. Сплетение $H ح K$ двух конечных неединичных групп $H$ и $K$ является 2-рефлексивной группой тогда и только тогда, когда $H$ - 2-рефлексивная группа, а $K$ - элементарная абелева 2-группа.

Прежде чем приступить к доказательству теоремы, отметим одно свойство 2рефлексивных групп, которым будем пользоваться в дальнейшем.

Пусть $G$ - группа, $a, b$ - инволюции из $G$ и $g=a$ или $g=a b$. Тогда $g^{a}=$ $g^{-1}$. Наоборот, если $g \in G$ и существует такая инволюция $a \in G$, что $g^{a}=g^{-1}$, то $g=(g a) \cdot a$ и $(g a)^{2}=1$. Таким образом, условие 2 -рефлексивности группы $G$ эквивалентно тому, что любой ее элемент инвертируется некоторой инволюцией. 
ДоКАЗАТЕЛЬСТвО ТЕОРЕМЫ 2. Необходимость условий, сформулированных в теореме 2, доказывается так же, как и в теореме 1.

Докажем их достаточность. Пусть $H$ является 2-рефлексивной группой, $K$ элементарной абелевой 2-группой и пусть $k_{1}, \ldots, k_{s}$ - множество всех различных элементов группы $K$. Для доказательства 2-рефлексивности $G$ нам достаточно показать, что каждый элемент из $G$ инвертируется некоторой инволюцией из $G$. Как и выше рассмотрим два возможных случая.

Случай 1. Пусть $g=h_{1}^{k_{1}} h_{2}^{k_{2}} \cdots h_{s}^{k_{s}}$, где $h_{1}, h_{2}, \ldots, h_{s} \in H$. Так как группа $H$ является 2-рефлексивной, то в $H$ найдутся такие инволюции $x_{1}, x_{2}, \ldots, x_{s}$, что $h_{1}^{x_{1}}=$ $h_{1}^{-1}, h_{2}^{x_{2}}=h_{2}^{-1}, \ldots, h_{s}^{x_{s}}=h_{s}^{-1}$. Пусть $x=x_{1}^{k_{1}} x_{2}^{k_{2}} \cdots x_{s}^{k_{s}}$. Тогда $x^{2}=1$ и

$$
g^{x}=\left(x_{1} h_{1} x_{1}\right)^{k_{1}}\left(x_{2} h_{2} x_{2}\right)^{k_{2}} \cdots\left(x_{s} h_{s} x_{s}\right)^{k_{s}}=g^{-1} .
$$

Случай 2. Как и в теореме 1 достаточно рассмотреть случай, когда $|K|=2$. Пусть $h, f \in H, k$ - неединичный элемент из $K$ и $g=k \cdot h f^{k}$. Определим элемент $z$ следующим образом. Положим $z=h f$, если $(h f)^{2}=1$. Если порядок элемента $h f$ больше двух, то полагаем $z=y^{k} h y f$, где $y$ - инволюция, инвертирующая элемент $h f$ (она существует, так как группа $H$ 2-рефлексивна). Пусть $x=z^{k}$. Очевидно, что $x^{2}=1$. Покажем, что $g^{x}=g^{-1}$. Имеем

$$
\begin{gathered}
g^{-1}=k \cdot f^{-1}\left(h^{-1}\right)^{k} \\
g^{x}=z^{k} \cdot\left(k \cdot h f^{k}\right) \cdot z^{k}=k \cdot z h f^{k} z^{k}=k \cdot z h(f z)^{k} .
\end{gathered}
$$

Следовательно, для доказательства равенства $g^{x}=g^{-1}$ нам достаточно показать, что

$$
z h(f z)^{k}=f^{-1}\left(h^{-1}\right)^{k} .
$$

Рассмотрим три возможных случая:

а) $h f=1$,

б) $|h f|=2$,

в) $|h f|>2$.

Если выполняется условие а), то $x=1$ и $h=f^{-1}$. Отсюда

$$
z h(f z)^{k}=h f^{k}=f^{-1}\left(h^{-1}\right)^{k} .
$$

Если элемент $h f$ является инволюцией, то

$$
h^{-1}=f h f \quad \text { и } \quad f^{-1}=h f h .
$$

Используя равенства (13), получаем

$$
z h(f z)^{k}=h f h(f h f)^{k}=f^{-1}\left(h^{-1}\right)^{k} .
$$

Предположим, что выполняется условие в). Тогда

$$
\begin{aligned}
z h(f z)^{k} & =\left(y^{k} h y f h\right)\left(f y^{k} h y f\right)^{k}=(h y f h y)(y f h y f)^{k} \\
& =\left(h(f h)^{-1}\right)\left((f h)^{-1} f\right)^{k}=f^{-1}\left(h^{-1}\right)^{k} .
\end{aligned}
$$

Таким образом, равенство (12) справедливо во всех случаях. Значит, $g^{x}=g^{-1}$. Теорема доказана. 
О РАЦИОНАЛЬНОСТИ И 2-РЕФЛЕКСИВНОСТИ СПЛЕТЕНИЙ КОНЕЧНЫХ ГРУПП

3. Приложение к классическим линейным группам. Доказанные в предыдущих разделах критерии рациональности и 2-рефлексивности сплетения двух конечных групп позволяют ответить на следующий вопрос: силовские 2-подгруппы каких классических линейных групп над конечным полем нечетной характеристики являются 2-рефлексивными, а каких - рациональными? Справедлива

Tеорема 3. Пусть $G$ - одна из классических линейнъх групn $G L_{n}(q), S p_{2 n}(q)$, $U_{n}(q), O_{2 n+1}^{+}(q) O_{2 n}^{ \pm}(q)$ над полем нечетной характеристики и $S$ - ее силовская 2-подгруппа. Тогда

1) $S$ является 2-рефлексивной тогда и только тогда, когда $G$ ортогональная групnа $O_{2 n+1}^{+}(q)$ или $O_{2 n}^{ \pm}(q)$;

2) $S$ - рациональна тогда и только тогда, когда $G=S p_{2 n}(q), O_{2 n+1}^{+}(q), O_{2 n}^{ \pm}(q)$ $u\left(q^{2}-1,16\right)=8$.

ДокАЗАТЕльство. Согласно [9] группа $S$ раскладывается в прямое произведение сплетений вида $\left(\ldots\left(W>Z_{2}\right) \imath \ldots\right)$ 乙 $Z_{2}$, в каждом из которых число сомножителей $Z_{2}$ и структура подгруппы $W$ определяется типом группы $G$. Исключение составляет лишь случай $G=O_{2 n}^{ \pm}(q)$, когда прямое произведение может содержать еще сомножитель вида $Z_{2} \times Z_{2}$. Таким образом, чтобы доказать теорему 3 нам достаточно, в силу теорем 1 и 2, ответить на следующий вопрос: в каких случаях группа $W$ является 2-рефлексивной, а когда - рациональной?

Напомним строение 2-группы $W$. Обозначим через $s$ наибольший целый показатель степени числа 2 , делящей $q^{2}-1$. Хорошо известно [9], что группа $W$ изоморфна обобщенной группе кватернионов порядка $2^{s}$, если $G=S p_{2 n}(q)$, и циклической или полудиэдральной группам порядков $\geqslant 4$ и $\geqslant 16$ соответственно, когда $G=G L_{n}(q)$ или $G=U_{n}(q)$. Наконец, если $G$ ортогональная группа $O_{2 n+1}^{+}(q)$ или $O_{2 n}^{ \pm}(q)$, то $W$ изоморфна диэдральной группе порядка $2^{s}$.

Очевидно, что циклическая группа порядка $2^{t}$ является рациональной (2-рефлексивной) тогда и только тогда, когда $t=1$, а в полудиэдральной группе

$$
\left\langle x, y \mid x^{2^{t+1}}=1, x^{2^{t}}=y^{2}, x^{y}=x^{2^{t}-1}\right\rangle
$$

порядка $2^{t+2} \geqslant 16$ элементы $x$ и $x^{-1}$ не сопряжены, поэтому она не является ни рациональной, ни 2-рефлексивной. Отсюда следует, что силовские 2-подгруппы групп $G L_{n}(q)$ и $U_{n}(q)$ не являются ни рациональными, ни 2-рефлексивными.

Далее, диэдральная группа

$$
\left\langle x, y \mid x^{2^{s-1}}=y^{2}=1, x^{y}=x^{-1}\right\rangle
$$

порядка $2^{s} \geqslant 4$ является 2-рефлексивной при любом $s$, а рациональной - только, если $s=2,3$. Наконец, обобщенная группа кватернионов

$$
\left\langle x, y \mid x^{2^{s-1}}=1, x^{2^{s-2}}=y^{2}, x^{y}=x^{-1}\right\rangle
$$

порядка $2^{s} \geqslant 4$ не является 2-рефлексивной, так как в ней элементы $x$ и $x^{-1}$ сопряжены не инволюцией, а элементом порядка 4. Рациональной она является тогда и 
только тогда, когда $s=2,3$. В заключении остается заметить, что при нечетном $q$ число $q^{2}-1$ всегда делится на 8 . Теорема доказана.

\section{СПИСОК ЦИТИРОВАННОЙ ЛИТЕРАТУРЫ}

[1] "Коуровская тетрадь", Нерешенные вопросы теории групn, 15-е изд., ИМ СО РАН, Новосибирск, 2002.

[2] Ж.-П. Серр, "Группы Галуа над $\mathbb{Q} "$, Труды семинара Н. Бурбаки за 1988 г., Математика. Новое в зарубежной науке, 46, Мир, М., 1990.

[3] А.И. Кострикин, И. А. Чубаров, "Представление конечных групп", Итоги науки и техники. Алгебра, геометрия и топология. Фундамент. направления, 23, ВИНИТИ, M., 1985, 119-196.

[4] С. Г. Колесников, "О рациональности значений комплексных характеров силовских 2-подгрупп симметрических групп $S_{2}$ ", Algebra and Model Theory, 4, НГТУ, Новосибирск, 2003, 44-46.

[5] М. Холл, Теория групп, ИЛ, М., 1962.

[6] G. Baumslag, "Wreath products and p-groups", Proc. Cambridge Philos. Soc., 55 (1959), 224-231.

[7] В.Н. Ремесленников, "Финитная аппроксимируемость групп относительно сопряженности", Сиб. матем. ж., 12:5 (1971), 1085-1099.

[8] Ф. Бахман, Построение геометрии на основе понятия симметрии, Наука, М., 1969.

[9] R. Carter, P. Fong, "The Sylow 2-subgroups of the finite classical groups", J. Algebra, 1:2 (1964), 139-151.

\section{С. Г. Колесников}

Поступило

Красноярский государственный университет

21.03.2005

E-mail: sklsnkv@mail.ru

Исправленный вариант

20.09.2005 